\title{
Lichtaktivierte Sensoren zur empfindlichen Amindetektion
}

\author{
Virginia Valderrey ${ }^{+}$, Aurelio Bonasera ${ }^{+}$, Sebastian Fredrich und Stefan Hecht*
}

\begin{abstract}
Unsere neue, einfache und akkurate colorimetrische Methode basiert auf Diarylethenen (DAEs) zur schnellen Detektion einer großen Vielfalt primärer und sekundärer Amine. Die Sensoren bestehen aus aldehyd-oder ketonsubstituierten Diarylethenen, die selektiv ausgehend vom geschlossenen Isomer eine amininduzierte Entfärbungsreaktion eingehen. Somit können diese Sensoren zum gewünschten Zeitpunkt durch Lichteinstrahlung aktiviert werden und erlauben eine Empfindlichkeit der Amindetektion bis hinab zu $10^{-6} \mathrm{M}$ in Lösung. Zusätzlich ermöglicht die Immobilisierung auf Papier den Nachweis biogener Amine wie Cadaverin in der Gasphase oberhalb eines Grenzwerts von 12 ppbv innerhalb von $30 \mathrm{Se}$ kunden.
\end{abstract}

$\boldsymbol{A}_{\text {mine sind allgegenwärtige natürliche Bestandteile aller }}$ lebenden Individuen (z. B. Aminosäuren, Neurotransmitter), und einige von ihnen können oberhalb bestimmter Konzentrationen Anzeichen für industrielle Verschmutzung und verdorbene Lebensmittel sein. ${ }^{[1]}$ Verschiedenste biogene Amine wie Cadaverin, Histamin, Tyramin, Putrescin, Spermidin, Spermin und Ethanolamin sind die Produkte thermischer oder enzymatischer Decarboxylierung von Aminosäuren durch Bakterien. ${ }^{[2]}$ Daher kann die Anreicherung dieser Amine als Indikator für Lebensmittelqualität oder Hygiene fungieren. ${ }^{[3]}$ Die Detektion und Unterscheidung von Substanzen, die mit einer vorliegenden Umweltgefährdung in Zusammenhang gebracht werden können, bleibt eine stete Herausforderung für die Wissenschaft, weshalb der Nachweis von Aminen weitreichend untersucht wird und bereits viele Verfahren zur Detektion beschrieben wurden. Dabei werden bevorzugt mit Massenspektrometrie gekoppelte chromatographische Techniken genutzt, da sie gute Nachweisgrenzen für Amine liefern. ${ }^{[4]}$ Allerdings bleibt ihre Praktikabilität durch ihre ausgedehnten Analysezeiten sowie die teure und platzraubende Gerätetechnik oft eingeschränkt. Antikörper und Enzyme liefern ebenfalls gute Empfindlichkeiten, ${ }^{[5]}$ haben aber den Nachteil, dass sie Materialien nutzen, die empfindlich gegen Chemikalien und die Temperatur sind. Zudem müssen Antikörper erst aufgezogen werden, was einen limitierenden Faktor für die Verfügbarkeit des aktiven Materials darstellt. Zur Überwindung dieser Hindernisse wurden bereits vielfältige colorimetrische, auf einfachen

[*] Dr. V. Valderrey, ${ }^{[+]}$Dr. A. Bonasera, ${ }^{[+]}$S. Fredrich, Prof. S. Hecht Institut für Chemie \& IRIS Adlershof

Humboldt-Universität zu Berlin

Brook-Taylor-Straße 2, 12489 Berlin (Deutschland)

E-Mail: sh@chemie.hu-berlin.de

$\left.{ }^{+}\right]$Diese Autoren haben gleichwertig zu diesem Artikel beigetragen.

(1) Hintergrundinformationen (Details zur Synthese und Charakterisie-

(D) rung) und die Identifikationsnummer (ORCID) eines Autors sind unter http://dx.doi.org/10.1002/ange.201609989 zu finden.
Chromophoren basierende Sensoren entworfen, die Amine über nichtkovalente Wechselwirkungen erkennen. Dazu wurden verschiedenste Konzepte der Aminsensorik entwickelt, wie die colorimetrischen Arrays von Suslick et al., ${ }^{[6]}$ die Polythiophencarbonsäuren von Lavigne et al., ${ }^{[7]}$ die Rezeptoren von Anslyn et al. ${ }^{[8]}$ der Cumarin-Test von Severin et al. ${ }^{[9]}$ das Cruciform von Miljanić und Lim $^{[10]}$ sowie der Cyclodextrinfarbstoff von Kaneda et al. ${ }^{[11]}$ Es wurde weiterhin ein colorimetrischer Sensor für Amine beschrieben, der Enzyme und Chromophore miteinander verbindet. ${ }^{[12]}$ Kürzlich wurden photochrome Materialien als Sensoreinheiten für die Detektion verschiedener Analyten eingesetzt, darunter Aminosäuren, Cyanidanionen, Thiole oder Organophosphorverbindungen. ${ }^{[13]}$ In diesen Beispielen kann die Reaktivität des Chromophors durch Bestrahlung mit UV- oder sichtbarem Licht beeinflusst werden. Dieser Ansatz ist geeigneter für praktische Anwendungen, da der Sensor in seiner ruhenden Form bis zur Aktivierung des Moleküls kurz vor seinem Einsatz aufbewahrt werden kann. Darüber hinaus beeinträchtigt eine Exposition des inaktiven Sensors gegenüber einem aminhaltigen Milieu nicht die Leistungsfähigkeit der aktiven Spezies, wodurch eine Überbestimmung der Analytdetektion vermieden werden kann. Hier beschreiben wir eine Methode für die effektive Erkennung und Quantifizierung von Aminen mittels Diarylethenen (DAEs), die zum gewünschten Zeitpunkt und in definierten Bereichen auf einer Oberfläche aktiviert werden kann, indem Licht als Fernsteuerung genutzt wird, ohne die lokale Konzentration zu beeinflussen.

Im Allgemeinen gehen DAEs eine $6 \pi$-Elektrocyclisierung und eine Cycloreversion unter Bestrahlung mit UV- bzw. sichtbarem Licht ein. ${ }^{[14]}$ Diese photochemische Reaktion geht mit einer reversiblen Änderung der sichtbaren Farbe einher: von der des offenen Isomers, das im nahen UV-Bereich absorbiert, hin zu jener der deutlich rotverschobenen, geschlossenen Form. Hinzu kommt eine durch die Photoisomerisierung hervorgerufene elektronische Veränderung, die eine Kontrolle über die Reaktivität geeignet substituierter Diarylethene, wie aldehydsubstituierter DAEs in Kondensationsreaktionen mit Aminen, ermöglicht. ${ }^{[15]}$ Angeregt durch diese Beispiele, wollten wir DAEs mit Carbonylgruppen am Ende des Hexatriensystems herstellen, die eine noch deutlichere Veränderung der Reaktivität gegen Amine aufweisen sollten. Dafür synthetisierten wir eine Serie photoschaltbarer Diarylethene, die an der reaktiven Position eines der Heteroarene mit einer Formyl- (10-3o) oder Acetylgruppe (4o) substituiert waren (Schema 1). Der Ringschluss der offenen DAEs (1o-4o) mit UV-Licht wandelt die weniger reaktiven aromatischen nun in reaktivere aliphatische Carbonylgruppen um. $^{[16]}$

Aus der UV/Vis-Absorptionsspektroskopie einer $4 \times 10^{-5} \mathrm{M}$ Lösung von $1 \mathrm{o}$ ist zu entnehmen, dass das offene 


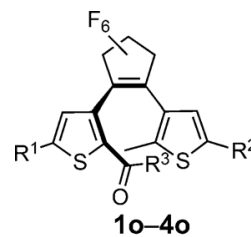

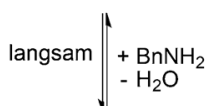<smiles>[R]c1cc2c3c(/c(=N\Br)c4sc([R])cc4/c(=N\C)c2s1)CCC3</smiles>

1io-4io

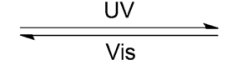

amininduzierte Umlagerung
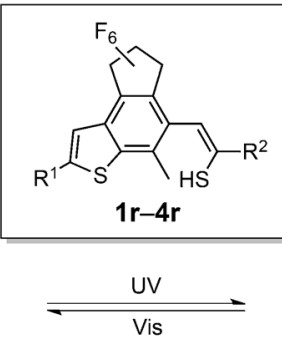
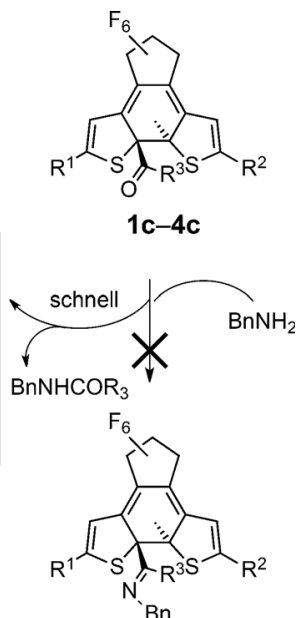

1ic-4ic

2: $R^{1}=4-O M e-P h, R^{2}=P h, R^{3}=H$
ing

3: $R^{1}=P h, \quad R^{2}=P h, R^{3}=H$

4: $\mathrm{R}^{1}=\mathrm{Ph}, \quad \mathrm{R}^{2}=\mathrm{Ph}, \mathrm{R}^{3}=\mathrm{CH}_{3}$

Schema 1. Licht kontrolliert die Reaktivität von offenen (10-4o) und geschlossenen (1 c-4c) Diarylethenisomeren mit aromatischen oder aliphatischen Aldehyd-/Ketongruppen gegen Amine entweder zu Iminen (1 $\mathbf{i}-\mathbf{4} \mathbf{i})$ oder zu Benzothiophen-Umlagerungsprodukten (1 r-4 r). o: offen (open), c: geschlossen (closed), i: Imin (imine), r: umgelagert (rearranged). $\mathrm{Bn}=$ Benzyl, TMS $=$ Trimethylsilyl.

Isomer 10 bei Bestrahlung mit Licht der Wellenlänge $\lambda_{\text {irr }}=$ $365 \mathrm{~nm}$ eine Elektrocyclisierung eingeht (siehe Abbildung S10 in den Hintergrundinformationen). Dies wird deutlich durch die Bildung einer Bande im sichtbaren Bereich $\left(\lambda_{\max }=564 \mathrm{~nm}\right)$, begleitet von einer Farbänderung von farblos nach violett. Eine UPLC-Analyse der bestrahlten Lösung zeigt, dass im photostationären Zustand (PSS) etwa $80 \%$ von 1 o zum geschlossenen Isomer 1c umgesetzt wurden. Durch Bestrahlung einer Lösung von 1 im PSS mit $\lambda_{\text {irr }}=500 \mathrm{~nm}$ kann das reine offene Isomer 10 zurückgewonnen werden. Dieser Prozess kann über mehrere Zyklen wiederholt werden (siehe Abbildung S20; Abbildungen S15-S19 für thermische Stabilitäten). Die Reaktivität der beiden Isomere gegen Amine wurde zunächst mittels ${ }^{1} \mathrm{H}$-NMR-Spektroskopie untersucht (Abbildung 1, unten). Durch Bestrahlung einer farblosen millimolaren Lösung von 10 mit Licht der Wellenlänge $\lambda_{\text {irr }}=$ $350 \mathrm{~nm}$ über $30 \mathrm{~min}$ entsteht eine tiefviolette Lösung, deren ${ }^{1}$ H-NMR-Spektrum die typischen Signale des offenen und geschlossen Isomers in einem Verhältnis aufweist, das demjenigen des durch UPLC bestimmten PSS für die $4 \times 10^{-5} \mathrm{M}$ Lösung nahe kommt. Versetzen der bestrahlten Lösung mit 1.0 Äquiv. Benzylamin (BA) resultiert in einer unmittelbaren Entfärbung der Lösung.

Nach 30 min zeigt das ${ }^{1} \mathrm{H}-\mathrm{NMR}$-Spektrum die Signale des Protonensignals von 10 unverändert, was dafür spricht, dass das offene DAE-Isomer komplett unreaktiv gegenüber Aminen ist. Im Unterschied dazu verändern sich die Protonensignale des geschlossenen Isomers 1c. Das Aldehydsignal von $1 \mathbf{c}$ verschwindet, ein Hinweis darauf, dass die nukleophile Addition des Amins an der Aldehydgruppe selektiv im geschlossenen Isomer stattgefunden hat. Ähnliche Ergebnisse werden auch mittels UV/Vis-Absorptionsspektroskopie er-

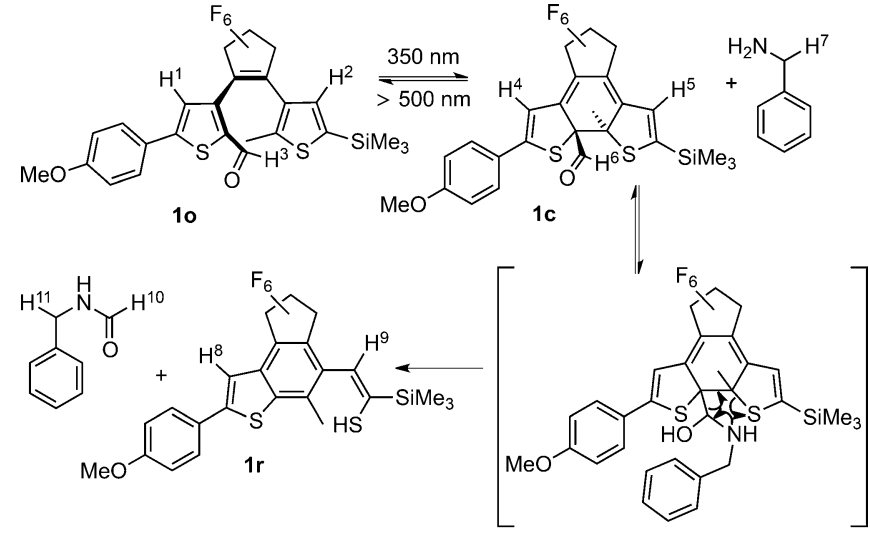

d) N-Benzylformamid

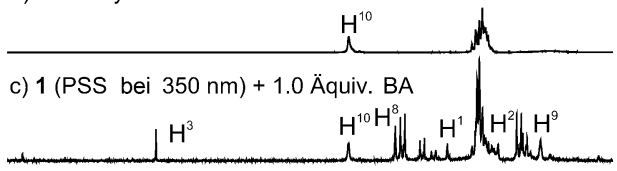

$\mathrm{H}^{11}$

b) 1 (PSS bei $350 \mathrm{~nm}, 30 \mathrm{~min}$ )
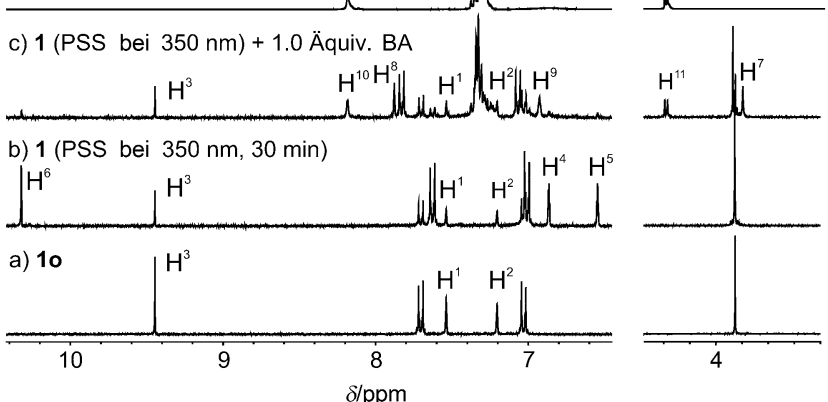

Abbildung 1. Oben: vorgeschlagener Mechanismus für die amininduzierte Entfärbung von aldehydsubstituierten DAEs. Unten: Ausgewählte Regionen aus den ${ }^{1} \mathrm{H}-N M R-S p e k t r e n\left(\mathrm{CD}_{3} \mathrm{CN}, 298 \mathrm{~K}\right)$ von a) 10 ; b) einer Mischung aus 10 und $1 \mathrm{c}$ entsprechend dem PSS nach 30minütigem Bestrahlen mit $\lambda_{\text {irr }}=350 \mathrm{~nm}$; c) einer Mischung aus 10 und $1 \mathrm{c}$ entsprechend dem PSS nach 30 minütigem Bestrahlen mit $\lambda_{\text {irr }}=350 \mathrm{~nm}+1.0$ Äquiv. BA; d) reinem $N$-Benzylformamid.

halten. So resultiert die Zugabe von BA zu einer Lösung aus 1o, bestrahlt mit $\lambda_{\text {irr }}=365 \mathrm{~nm}$ zum Erreichen des PSS, in einer sukzessiven Abnahme der Bande bei $\lambda_{\max }=564 \mathrm{~nm}$ im sichtbaren Bereich des Spektrums, einer hypsochromen Verschiebung der Bande im UV-Bereich und somit einer gelblichen Färbung der Lösung (Abbildung 2a). Sobald keine weitere Veränderung der Absorptionsspektren mehr stattfand, wurde die Lösung erneut mit $\lambda_{\text {irr }}=365 \mathrm{~nm}$ bestrahlt; allerdings konnten keine weiteren spektralen Veränderungen mehr erhalten werden. In Anbetracht dieser Ergebnisse schlussfolgern wir, dass 1c selektiv in Gegenwart von 10 irreversibel mit BA zu einem photoinaktiven Produkt $1 \mathbf{r}$ reagiert. Um die Struktur von 1r aufzuklären, isolierten wir das geschlossene Isomer 1c und setzten es mit 1.0 Äquiv. BA um (weitere Details in den Hintergrundinformationen). Die Röntgendiffraktometrie der reinen Substanz 1r zeigt nun, dass sich die zentrale DAE-Einheit infolge des Abbaus des dem Aldehyd gegenüberliegenden Thiophenrings $\mathrm{zu}$ einem Benzothiophengerüst umlagert ${ }^{[17]}$ und schließlich eine photochemisch unreaktive Verbindung ergibt (Abbildung 2b).

Das Auftreten von $N$-Benzylformamid als Nebenprodukt im ${ }^{1} \mathrm{H}$-NMR-Experiment liefert weitere Erkenntnisse über einen sinnvollen Mechanismus der beobachteten Umlagerung. Zuerst findet ein nukleophiler Angriff des Amins am 


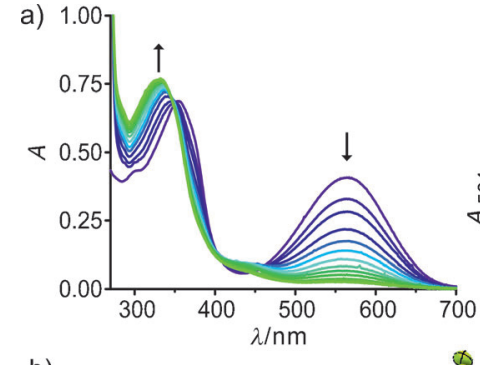

b)
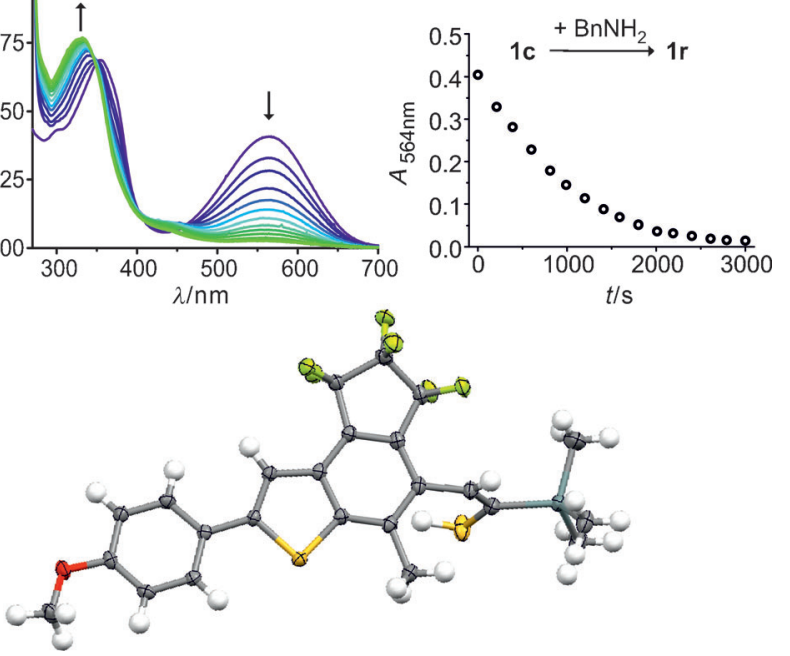

Abbildung 2. a) Links: Entwicklung der UV/Vis-Absorptionsspektren für den PSS von $1\left(2.4 \times 10^{-5} \mathrm{M}\right)$ nach Zugabe von BA $\left(1.7 \times 10^{-2} \mathrm{M}\right)$. Rechts: Die Abnahme der Bande bei $\lambda_{\max }=564 \mathrm{~nm}$ entsprechend der Bildung von 1 r. b) Röntgenkristallstruktur von 1 r; grau $\mathrm{C}$, weiß $\mathrm{H}$, grün $\mathrm{F}$, rot $\mathrm{O}$, gelb $\mathrm{S}$. Die Ellipsoide repräsentieren $50 \%$ Aufenthaltswahrscheinlichkeit. Die Wasserstoffatome sind als Kugeln mit willkürlichem Radius dargestellt. ${ }^{[18]}$

hochreaktiven aliphatischen Aldehyd des geschlossenen Isomers statt und bildet das entsprechende Halbaminalderivat. Anschließend lagert sich dieses Derivat unter Eliminierung von $N$-Benzylformamid zur stabileren Verbindung 1r um (Abbildung 1, oben). Zur Untermauerung des vorgeschlagenen Mechanismus der Bildung von 1r synthetisierten wir das Iminderivat $1 \mathbf{i}$ ausgehend vom offenen Diarylethen 1o. Wie erwartet kann 1i zwischen seinem offenen und geschlossenen Isomer unter abwechselnder Bestrahlung mit UV- und sichtbarem Licht reversibel schalten, ohne 1r zu bilden (Schema 1 und Abbildung S35). Dies steht in Übereinstimmung mit dem vorgeschlagenen Mechanismus zur Bildung von 1r, der notwendigerweise über das Halbaminalderivat verlaufen muss (Abbildung 1, oben). Vergleichbare Experimente wurden auch mit den Schaltern 2-4 durchgeführt. Die UV/ Vis-Absorption sowie die ${ }^{1} \mathrm{H}-\mathrm{NMR}$-Spektren bestätigen die Umsetzung der untersuchten Schalter zu Produkten, die dieselben zentralen Benzothiophengerüste aufweisen wie 1r und sich nur in ihren Substitutionsmustern unterscheiden (weitere Details siehe Hintergrundinformationen). Der entscheidende Unterschied zwischen den amininduzierten Umlagerungen der DAEs 1c-4c ist deren Reaktionsgeschwindigkeit, die die Reaktivität der Carbonyleinheit widerspiegelt. Wie erwartet folgt die Reaktivität der Reihe: $\mathbf{3 c}>\mathbf{1} \mathbf{c} \approx$ 2c $>$ 4c (UV/Vis-Spektren siehe Hintergrundinformationen). Diese Reihenfolge kann damit erklärt werden, dass das weniger reaktive Keton $\mathbf{4} \mathbf{c}$ das Halbaminalintermediat deutlich langsamer bildet als die Aldehyde, von denen $\mathbf{3 c}$ das schnellste ist, da es keine elektronenschiebende Methoxygruppe trägt. Man beachte, dass der Effekt der Substitution am Thiophenring geringer ist der einer leichten Veränderung der Aminstruktur, was den hohen Grad an Chemoselektivität für die Bestimmung der Amine verdeutlicht.
Nach der unerwarteten Entdeckung dieser spezifischen Umlagerung der geschlossenen, carbonylsubstituierten DAEIsomere haben wir eine systematische spektroskopische Untersuchung vorgenommen, um 1) die Geschwindigkeitskonstanten für die Reaktion aller vier Schalter mit einer Vielfalt an Aminen zu quantifizieren und 2) die geringste Konzentration zu bestimmen, für die eine Entwicklung der spektralen Banden innerhalb einer sinnvollen Zeitspanne abläuft (d.h. bis zu $2 \mathrm{~h}$ ).

Es wurde eine Bibliothek von acht Aminen in Kombination mit den synthetisierten DAE-Schaltern untersucht (Tabelle 1 und Hintergrundinformationen). Die Amine wurden

Tabelle 1: Geschwindigkeitskonstanten erster Ordnung $(k)$, ermittelt für die Umlagerungsreaktion der DAEs $1 \mathrm{c}$ und $3 \mathrm{c}$ mit verschiedenen Aminen.

\begin{tabular}{|c|c|c|c|}
\hline $\operatorname{Amin}^{[a]}$ & Struktur & $k(1 c) / \min ^{[b]}$ & $k(3 c) / \min ^{[b]}$ \\
\hline SPER & & 1.67 & 2.66 \\
\hline CAD & $\mathrm{H}_{2} \mathrm{~N}^{-}$ & 0.30 & 0.50 \\
\hline DAP & $\mathrm{H}_{2} \mathrm{~N}^{-}$ & 0.49 & 1.58 \\
\hline OA & $\mathrm{H}_{2} \mathrm{~N}^{-}$ & 0.12 & 0.13 \\
\hline BA & $\mathrm{H}_{2} \mathrm{~N}^{-}$ & 0.033 & 0.025 \\
\hline MBA & $\mathrm{H}_{2} \mathrm{~N}^{-}$ & 0.002 & 0.0012 \\
\hline BMA & $\mathrm{H}^{-}$ & 0.0010 & 0.0007 \\
\hline DMBA & ${ }_{l}^{\mathrm{N}}$ & k.r. & k.r. \\
\hline
\end{tabular}

[a] $1 \times 10^{-2} \mathrm{M}$ in $\mathrm{CH}_{3} \mathrm{CN}$. [b] $2 \times 10^{-5} \mathrm{M}$ in $\mathrm{CH}_{3} \mathrm{CN}$.

so ausgewählt, dass sie repräsentativ verschiedene Klassen abdecken, wie tertiäre Amine ( $N, N$-Dimethylbenzylamin (DMBA $)$, sekundäre Amine (N-Benzylmethylamin (BMA)), voluminöse primäre Amine (Benzylamin und $(S)$ (-)- $\alpha$-Methylbenzylamin (MBA)), primäre lineare aliphatische Amine (Octylamin (OA)), primäre Diamine (1,3-Diaminopropan (DAP) und Cadaverin (CAD)) und aliphatische Triamine (Spermidin (SPER)). In einem typischen Experiment wurde eine $2 \times 10^{-5} \mathrm{M}$ Lösung des DAE bis zum Erreichen des PSS bestrahlt (PSS-Werte der verschiedenen Schalter: siehe Tabelle 2 in den Hintergrundinformationen). Danach wurden spezifische Mengen eines Amins zugesetzt. Wir verfolgten die Veränderung der optischen Dichte am Maximum der zugehörigen sichtbaren Bande des geschlossenen Isomers. Dieser Bereich wurde gewählt, weil die Absorption des Umlagerungsproduktes $1 \mathbf{r}-\mathbf{4} \mathbf{r}$ dort minimal und daher der Unterschied der Absorption maximal ist. Über ein Angleichen der spektralen Veränderungen im UV/Vis-Spektrum nach einer Kinetik erster Ordnung wurden die Geschwindigkeitskonstanten bestimmt. Wie aus dem vorgeschlagenen Mechanismus zu erwarten war, sind die tertiären Amine nicht reaktiv. Sekundäre Amine reagieren deutlich langsamer als primäre, wie aus den Geschwindigkeiten von BMA verglichen mit denen von BA zu entnehmen ist. Diese Differenzierung entsteht vermutlich durch den erhöhten sterischen Anspruch der sekundären Amine. Aus ähnlichen Gründen reagiert BA schneller als MBA. Rein aliphatische Amine sind um etwa eine Größenordnung reaktiver als BA. 
Schlussendlich reagieren aliphatische Oligoamine wie DAP, CAD und SPER schneller, als aus der einfachen Korrektur der Anzahl der Aminogruppen in den entsprechenden Molekülen zu erwarten wäre. Offenbar scheinen Nachbargruppeneffekte hier eine maßgebliche Rolle zu spielen.

Bei der Betrachtung dieser Reaktivitätsdaten sollte insbesondere 3c, das bessere Eigenschaften als Photoschalter aufweist (hoher Anteil des geschlossenen Isomers im PSS), eine exzellente thermische Stabilität aufweist (siehe Abbildung S18 in den Hintergrundinformationen) und eine höhere Reaktivität gegen Amine zeigt, besondere Beachtung geschenkt werden. Laut den für CAD erhaltenen Daten findet die Umlagerung von 3c etwa 1.6-mal schneller statt als die von 1 c. Dabei wurde $2.0 \times 10^{-5} \mathrm{M}$ als unteres Konzentrationslimit festgestellt, für das $50 \%$ Abnahme der Absorption bei $\lambda_{\max }=580 \mathrm{~nm}$ innerhalb von $110 \mathrm{~min}$ erhalten wurde (siehe Abbildung S57).

Um die Bedingungen für die Aminbestimmung zu optimieren, untersuchten wir den Einfluss des $\mathrm{pH}$-Werts auf die Kinetik der Reaktion (siehe Abschnitt 6.1 in den Hintergrundinformationen). Die optimale Reaktionsgeschwindigkeit wurde nach Zugabe von zehn Äquivalenten $p$-Toluolsulfonsäure zu einer Lösung von $\mathbf{3 c}$ in Acetonitril, gefolgt von der Zugabe von zehn Äquivalenten CAD, erhalten. Daraus ergab sich eine 40-fache Steigerung der Geschwindigkeit gegenüber jener in Abwesenheit des Säurekatalysators. Diese Erkenntnis entspricht dem klassischen Szenario eines optimalen, leicht sauren $\mathrm{pH}-\mathrm{Fensters},{ }^{[19]}$ in dem kleine Mengen Säure die Elektrophilie der Carbonylkomponente erhöhen, zu viel Säure hingegen zur Protonierung des Amins führt, wodurch dessen Nukleophilie reduziert wird.

Nachdem die Reaktionsbedingungen für die Aminbestimmung optimiert worden waren, konzentrierten wir uns auf die Anwendung dieses Verfahrens unter Nutzung einer einfachen und preiswerten Plattform. Eingangs entschieden wir uns, einen Farbcode-Assay zu erstellen, der es ermöglicht, Amine mit bloßem Auge schnell und einfach zu detektieren. Verschiedene Mengen des Amins wurden zu einer Mikrotiterplatte mit 16 Kavitäten gegeben, die mit einer Lösung aus 3 gefüllt waren (Acetonitril, $\lambda_{\text {irr }}=365 \mathrm{~nm}$ ). Als Ergebnis wurde die charakteristische Farbveränderung von tiefviolett nach gelb mit verschiedenen Geschwindigkeiten erhalten. Die Manipulation von während des Versuchs aufgenommenen Bildern der Platte ermöglichte es uns, eine Farbpalette anzufertigen, die genutzt werden kann, um die Aminkonzentration in Lösung innerhalb kurzer Zeit bestimmen zu können (siehe Abbildungen S62 und S63).

Um die Vielseitigkeit unserer Nachweisstrategie aufzuzeigen, erweiterten wir unsere Studien in Lösung um die Anfertigung aminempfindlicher, fester Substrate. Ein Filterpapier wurde mit dem farblosen, inaktiven DAE 3o beschichtet (Abbildung 3a). Danach wurde die Oberfläche mit einer Photomaske bedeckt und mit $\lambda_{\text {irr }}=365 \mathrm{~nm}$ bestrahlt. Somit konnten wir durch Umsetzung von 30 in die aktive Form 3c ein Farbmuster in gewünschten, präzisen Bereichen der Oberfläche generieren (Abbildung $3 \mathrm{~b}$ ). Anschließend setzten wir die Probe Dämpfen von CAD aus und beobachteten, dass nur jene Bereiche, die aktiven Sensor 3c enthielten, entfärbt wurden (Abbildung 3c). Darüber hinaus können

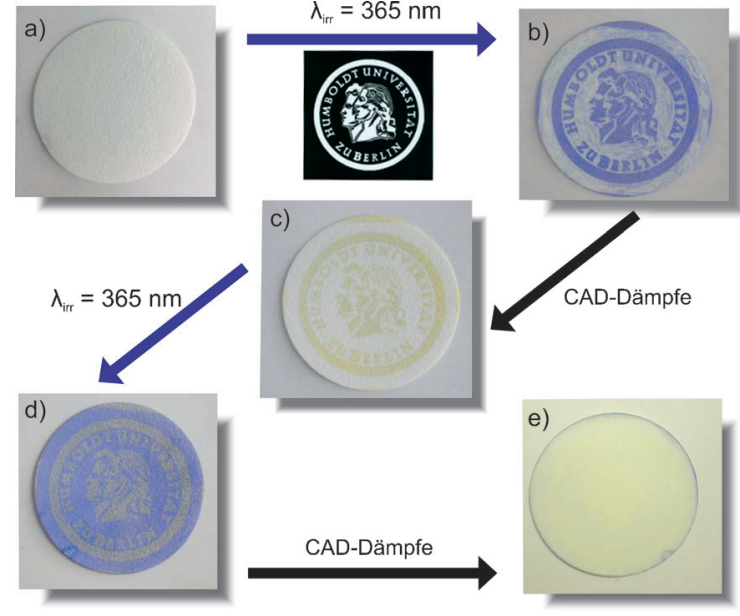

Abbildung 3. Aminbestimmung auf lichtaktiviertem Filterpapier. a) Mit einer Lösung von $\mathbf{3}$ o beschichtetes Filterpapier. b) In-situ-Aktivierung durch Bestrahlung bei $\lambda_{\text {irr }}=365 \mathrm{~nm}$ über 2 min mit einer UV-Lampe nach vorheriger Bedeckung der Oberfläche mit einer Photomaske (ausgedruckt mit einem herkömmlichen Laserdrucker auf transparenter Plastikfolie). c) Farbentwicklung nach Exposition des Filterpapiers gegenüber CAD-Dämpfen über 5 min. d) Nach Entfernen der CADDämpfe können die nichtbestrahlten Bereiche (übriges 3 o) durch 2minütige Bestrahlung mit $\lambda_{\text {irr }}=365 \mathrm{~nm}$ aktiviert werden und bilden so das Negativ zum Farbcode von (b). e) Farbentwicklung nach erneutem 5-minütigem Aussetzen des Filters gegenüber CAD-Dämpfen.

die Regionen, die zuvor nicht aktiviert worden waren, a posteriori aktiviert werden (Abbildung 3d). Dies ermöglicht es nicht nur, Strukturen darzustellen, sondern auch, den Sensor zur mehrfachen Nutzung schrittweise in kleinen Bereichen zu aktivieren.

Die sequenzielle UV-Aktivierung demonstriert gut, dass das inaktive Sensormaterial nicht mit Aminen reagiert, bis zu dem Moment, in dem es durch UV-Licht aktiviert wird, wodurch gesichert ist, dass der Sensor bis zum gewünschten Augenblick konserviert bleibt. Es ist hervorzuheben, dass es uns diese Methode ermöglicht, Cadaverindämpfe bis $\mathrm{zu}$ einem unteren Grenzwert von 12 ppbv (parts per billion by volume (Teile pro Milliarde in Volumen)) in $30 \mathrm{~s}$ zu bestimmen (siehe Abbildungen S65 und S66).

Zusammengefasst haben wir eine einfache Methode zur Aminbestimmung in Lösung und in der Gasphase entwickelt. Unser colorimetrischer Assay basiert auf aldehydsubstituierten DAEs, die durch UV-Licht aktiviert werden können. Nur das bei Bedarf generierte geschlossene Isomer kann eine Reaktion mit Aminen eingehen und führt zu einem entfärbten Umlagerungsprodukt (sowie einem Amid). Kinetische Studien über die einfache Verfolgung der UV/Vis-Absorption ermöglichten es uns, zwischen einer großen Vielfalt an Aminen zu differenzieren und schnelle Reaktionen in Gegenwart von primären aliphatischen Aminen von langsamer reagierenden sekundären Aminen zu unterscheiden, während tertiäre Amine überhaupt nicht reagierten. Gegenüber anderen Methoden auf Basis von Mehrkomponentensystemen oder bimolekularen Reaktionskaskaden, die zwei verschiedene empfindliche Moleküle voraussetzen, benötigt unsere Strategie nur eines. Hierbei finden die beiden sequenziellen 
Vorgänge gleichzeitig in derselben Sensoreinheit statt, was eine äußerst schnelle Rückmeldung liefert. Die geringe Reaktivität des offenen Isomers gegen Amine und die Möglichkeit, das DAE mittels Licht in definierten Bereichen auf Oberflächen zu aktivieren, machen es zum idealen Detektor für die Entwicklung einer neuen Generation von "intelligenten" und trotzdem preiswerten Sensoreinheiten. Aktuelle Arbeiten in unseren Laboren zielen auf:

1) eine gesteigerte Reaktivität der geschlossenen Form zur Umlagerung durch den Einbau elektronenziehender Substituenten, um die Thiolatfluchtgruppe zu stabilisieren (siehe Mechanismus in Abbildung 1, oben);

2) die Implementierung von Fluoreszenz als noch empfindlichere Auslesemethode;

3) einen gesteigerten photochemischen Umsatz im PSS im Sonnenlicht zum einfachen Einsatz in Entwicklungsländern.

Über die Aminbestimmung hinaus beabsichtigen wir, diese neue, vielversprechende Umsetzung zu einer lichtaktivierten Acyltransferreaktion für Biokonjugationen weiterzuentwickeln.

\section{Danksagung}

Wir danken Dr. Beatrice Cula Braun für die Röntgenkristallographiedaten und Michael Kathan für hilfreiche Diskussionen. Weiterhin danken wir dem ERC (ERC-2012STG_308117 "Light4Function"), der Europäischen Kommission (MSCA-ITN "iSwitch" GA Nr. 642196) und der DFG (SFB 658, Teilprojekt B8) sowie der Alexander von Humboldt-Stiftung.

\section{Interessenkonflikt}

Die Autoren erklären, dass keine Interessenskonflikte vorliegen.

[1] a) S. Bardócz, Trends Food Sci. Technol. 1995, 6, 341-346; b) A Naila, S. Flint, G. Fletcher, P. Bremer, G. Meerdink, J. Food Sci. 2010, $75, \mathrm{R} 139-\mathrm{R} 150$.

[2] a) B. ten Brink, C. Damink, H. M. L. J. Joosten, J. H. J. H. in’t Veld, Int. J. Food Microbiol. 1990, 11, 73-84; b) A. Halász, Á. Baráth, L. Simon-Sarkadi, W. Holzapfel, Trends Food Sci. Technol. 1994, 5, 42-49; c) M. H. S. Santos, Int. J. Food Microbiol. 1996, 29, 213-231.

[3] a) I. A. Bulushi, S. Poole, H. C. Deeth, G. A. Dykes, Crit. Rev. Food Sci. Nutr. 2009, 49,369-377; b) C. Y. Chong, F. Abu Bakar, A. R. Russly, B. Jamilah, N. A. Mahyudin, Int. Food Res. J. 2011 $18,867-876$
[4] a) D. C. Johnson, D. Dobberpuhl, R. Roberts, P. Vandeberg, J. Chromatogr. A 1993, 640, 79-96; b) S. Shesnov, L. Bigler, M. Hesse, Eur. J. Mass Spectrom. 2002, 8, 1-16; c) M. L. LatorreMoratalla, J. Bosch-Fusté, T. Lavizzari, S. Bover-Cid, M. T. Veciana-Nogués, M. C. Vidal-Carou, J. Chromatogr. A 2009, $1216,7715-7720$.

[5] a) J. Lange, C. Wittmann, Anal. Bioanal. Chem. 2002, 372, $276-$ 283; b) B. Bóka, N. Adányi, D. Virág, M. Sebela, A. Kiss, Electroanalysis 2012, 24, 181-186; c) S. Leonardo, M. Campàs, Microchim. Acta 2016, 183, 1881-1890.

[6] a) N. A. Rakow, A. Sen, M. C. Janzen, J. B. Ponder, K. S. Suslick, Angew. Chem. Int. Ed. 2005, 44, 4528-4532; Angew. Chem. 2005, 117, 4604-4608; b) J. R. Askim, M. Mahmoudi, K. S. Suslick, Chem. Soc. Rev. 2013, 42, 8649-8682.

[7] a) T. L. Nelson, C. O'Sullivan, N. T. Greene, M. S. Maynor, J. J. Lavigne, J. Am. Chem. Soc. 2006, 128, 5640-5641; b) M. S. Maynor, T. L. Nelson, C. O'Sulliva, J. J. Lavigne, Org. Lett. 2007, 9, 3217-3220; c) T. L. Nelson, I. Tran, T. G. Ingallinera, M. S. Maynor, J. J. Lavigne, Analyst 2007, 132, 1024-1030; d) M. Cai, S. L. Daniel, J. J. Lavigne, Chem. Commun. 2013, 49, 6504-6506.

[8] a) S. Nieto, J. M. Dragna, E. V. Anslyn, Chem. Eur. J. 2010, 16, 227-232; b) J. M. Dragna, G. Pescitelli, L. Tran, V. M. Lynch, E. V. Anslyn, L. Di Bari, J. Am. Chem. Soc. 2012, 134, 43984407; c) P. Metola, E. V. Anslyn, T. D. James, S. D. Bull, Chem. Sci. 2012, 3, 156-161; d) Y. T. Zhou, Y. L. Ren, L. Zhang, L. You, Y. F. Yuan, E. V. Anslyn, Tetrahedron 2015, 71, 3515-3521.

[9] B. Lee, R. Scopelliti, K. Severin, Chem. Commun. 2011, 47, 9639-9641.

[10] J. Lim, O. S. Miljanić, Chem. Commun. 2012, 48, 10301-10303.

[11] J. H. Jung, S. J. Lee, J. S. Kim, W. S. Lee, Y. Sakata, T. Kaneda, Org. Lett. 2006, 8, 3009-3012.

[12] P. Q. Leng, F. L. Zhao, B. C. Yin, B. C. Ye, Chem. Commun. 2015, 51, 8712-8714.

[13] a) N. Shao, J. Y. Jin, S. M. Cheung, R. H. Yang, W. H. Chan, T. Mo, Angew. Chem. Int. Ed. 2006, 45, 4944-4948; Angew. Chem. 2006, 118, 5066-5070; b) Y. Shiraishi, K. Adachi, M. Itoh, T. Hirai, Org. Lett. 2009, 11, 3482-3485; c) F. Nourmohammadian, T. Wu, N. R. Branda, Chem. Commun. 2011, 47, 10954-10956; d) Y. Shiraishi, K. Yamamoto, S. Sumiya, T. Hirai, Phys. Chem. Chem. Phys. 2014, 16, 12137-12142.

[14] a) M. Irie, Chem. Rev. 2000, 100, 1685-1716; b) H. Tian, S. J. Yang, Chem. Soc. Rev. 2004, 33, 85-97.

[15] a) D. Wilson, N. R. Branda, Angew. Chem. Int. Ed. 2012, 51, 5431-5434; Angew. Chem. 2012, 124, 5527-5530; b) M. Kathan, P. Kovaříček, C. Jurissek, A. Senf, A. Dallmann, A. F. Thünemann, S. Hecht, Angew. Chem. Int. Ed. 2016, 55, 13882-13886; Angew. Chem. 2016, 128, 14086-14090.

[16] C. Godoy-Alcántar, A. K. Yatsimirsky, J. M. Lehn, J. Phys. Org. Chem. 2005, 18, 979-985.

[17] Thermisch induzierte Umlagerung einiger Diarylethene zu Benzothiophenen: a) S. Kobatake, H. Imagawa, H. Nakatani, S. Nakashima, New J. Chem. 2009, 33, 1362-1367; b) D. Kitagawa, S. Kobatake, Chem. Lett. 2011, 40, 93-95; c) H. Shoji, S. Kobatake, Chem. Commun. 2013, 49, 2362-2364.

[18] CCDC 1508362 enthält die ausführlichen kristallographischen Daten zu dieser Veröffentlichung. Die Daten sind kostenlos beim Cambridge Crystallographic Data Centre erhältlich.

[19] R. G. Kallen, W. P. Jencks, J. Biol. Chem. 1966, 241, 5864-5878.

Manuscript received: October 12, 2016

Revised: November 18, 2016

Final Article published: 


\section{Zuschriften}

Colorimetrische Amindetektion

V. Valderrey, A. Bonasera, S. Fredrich,

S. Hecht*

III-IIII

Lichtaktivierte Sensoren zur empfindlichen Amindetektion

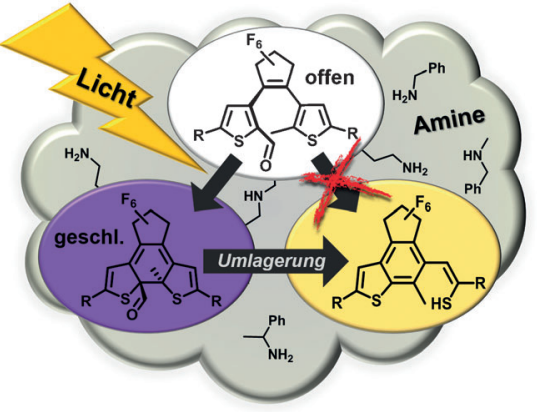

Farbe bekennen: Durch Aktivierung mit UV-Licht gehen Diarylethene mit internen Aldehydgruppen eine Umlagerung in Gegenwart von Aminen ein, die deren empfindliche colorimetrische Detektion ermöglicht (siehe Schema). 